We are informed that at Sir Thomas Maclear's funeral, on July 16 , all the principal residents in the colony were present. The Cape Parliament has passed a resolution or memorandum acknowledging the work he did for the colony.

\section{A POINT AFFECTING THE DIFFUSION OF THE GASES OF THE ATMOSPHERE IN RELATION TO HEALTH}

$\mathrm{THE}$ great importance in relation to bealth of the part played by the internal motion of gases, as indicated by the now established and admirably simple kinetic theory, would seem scarcely to receive adequate appreciation. The old and vaguely developed statical idea of a stagnant atmosphere with molecules at rest, has given place to the opposite view of a high activity of motion, even when the atmosphere appears to the senses to be still. By this motion noxious vapours or gases, instead of remaining stagnant, are rapidly scattered by diffusion, and thereby rendered harmless. The part apparently played here by inequality of molecular velocity (dependent on inequality of molecular mass) in contributing to this end, would seem scarcely to have received the attention it appears to deserve. In Prof. Tait's work, "Lectures on some Recent Advances in Physical Science" (p. 237, second edition), reference is made to the diffusion of the gases of the atmosphere under the kinetic theory, and here it would seem as if the influence of the inequality of the normal velocity of the molecules of the different gases of the atmosphere (dependent on inequality of molecular mass) had not been taken into account, and hence it would appear as if the gases in their mutual diffusion were regarded as subject to the pure contingencies of chance, as they would be if the velocities of the molecules were equal (or their masses equal); this necessarily leading to some rather startling conclusions, which make the continuance of life and health (as dependent on the equable mixture of the constituents of the atmosphere) a matter more or less dependent on contingency or accident. The passage in question runs as follows :-

"There is another extremely important point of this statistical question as to the particles of gases which I must carefully explain; and it is this, how it happens that in the enormous bulk of the whole atmosphere of the earth these particles of oxygen and nitrogen, moving about amongst one another, should not by chance, at some place or other, operate on one another in such a way that in some particular cubic inch the particles of nitrogen might for a moment expel from it all the particles of oxygen, so that in virtue of the great extent of the earth's atmosphere, compared with the size of a particle of gas, there might be at some definite instant a region filled mainly with nitrogen, and other regions filled mainly with oxygen. Now the beauty of this statistical method is that it explains to us how such an event, though perfectly possible, can never occur. It is a thing which is itself absolutely possible, but it never can occur in practice, because the probability of its occurrence is so exceedingly small. There is a probability (numerically measurable) for everything which is possible, but if that probability (reckoned in numbers) is as small as the probability of the accident we are crisiciering, we never expect to find it occur. And not $r$ iiy do we never expect to find it at any time, but we $c^{2}, 2$ say boldly from experience that it is never met with at all, however long our observations are conducted, or through however great an extent of space we conduct them. If you had originally in a box divided in to two equal parts, nitrogen in the one part and oxygen in the other, and then allowed them to mix with one another, the probability that in any assigned time you could find all the nitrogen back again in the space where it was originally, and all the oxygen back again in the space where it was originally, is certainly one which can be measured, but it is one which is so infinitesimally small that we know perfectly by experience that it can never be realised."

The above appears a somewhat unsatisfactory conclusion to contemplate, and there would seem to be something scarcely consistent in the inference that an event which is itself absolutely possible never can occur in practice, "because the probability of its occurrence is so exceedingly small." "For we know from the doctrine of probabilities that an event of chance (if possible at all) must occur, if the range of time be not restricted, or at least its probability approaches with indefinite closeness to absolute certainty in that case. That the probability, for example, of suffocation in a room [taking the above illustration of a box on a large scale] within a given range of time, by the oxygen separating itself sufficiently from the nitrogen, could be rigidly calculated, seems scarcely pleasant to contemplate, however remote the contingency might be, and it is hardly satisfactory to think that the contingency of such an event approaches with indefinite nearness to absolute certainty if an adequate time be conceded. The very fact that considering the vast extent of the atmosphere and the range of historic time, no record whatever exists of any irregularity having been detected in the constitution of the atmosphere, would surely be strong argument for the existence of some physical cause tending to prevent such irregularity from occurring, and removing it from the pure contingencies of chance. The above quotation that-" we can boldly say from experience that it $[i, e$. the irregularity] is never met with at all, however long our observations are conducted "-would surely tend to prove that some preventive means existed.

If the molecules of nitrogen and oxygen of a mass of air confined in a room were supposed subject to the pure contingencies of chance in their mutual actions in diffusion, they would be comparable to a number of equal perfectly elastic black and white balls imagined to be moving and colliding freely among themselves, or left to their own dynamics in an analogous manner. In this case there would evidently be practically an infinite number of chances against the molecules of the two gases (represented by the two differently coloured sets of balls) from becoming uniformly diffused through the room; indeed the probability of this event would be exactly the same as the probability of the oxygen being all separated in one part of the room and the nitrogen in the other (or in analogy all the black balls separated from the white); for we know that, according to the doctrine of probabilities, every assigned arrangement for all the balls is equally probable.

I venture to suggest that the inequality in the mean velocity of the molecules of the two gases (dependent on the inequality of the masses of the molecules) plays an important part here. If this particular point has been considered elsewhere (without my knowledge), I may still perhaps give an elementary analysis of the problem, as it has occurred to me. It may be remarked that on account of the simplicity of the kinetic theory, its problems frequently admit of elementary treatment, and it will at least be admitted that wherever this is practicable, perspicuity does not lose thereby. We will imagine for illustration a portion (say spherical shaped) of pure oxygen gas to be at a given instant of time surrounded by an atmosphere of hydrogen. [We may neglect the existence of gravity, for simplicity, as it does not affect the point with which we have to deal.] Then diffusion at once commences. The molecules of hydrogen which have one-sixteenth less mass, are known to possess a normal velocity four times that of the molecules of oxygen. The molecules of hydrogen by: their own normal motion will therefore rush into this spherical space occupied by the oxygen, four times as fast as the molecules of oxygen can move out by their natural motion. Owing to this inequality in the rate of exchange of places of the two gases, the mass of gas occupying the spherical space will begin to increase in density, and (for 
a converse cause) the gas surrounding the spherical space will diminish in density to a corresponding amount. This initial irregularity of density will cause an initial irregularity of pressure, which will tend at once forcibly to readjust itself, and will do so by the gaseous mixture within the spherical space expanding, ${ }^{1}$ and an exchange of energy (or "heat") taking place between the two gases-which abnormal state of things can only cẹse when the two gases become uniformly mixed, and consequently the dynamical conditions become symmetrical in all parts of the mixture. Owing to the absence of dynamical equilibrium in the case of two gases having different molecular velocities, unless the gases are uniformly mixed, there is therefore a forcible dynamical tendency to produce uniform mixture, and to maintain it against disturbing causes, when once the mixture has become uniform. When the molecules of the two gases possess unequal normal velocities (attendant on inequality of mass), it is evident sckat the distribution of velocities can be symmetrical throughout the mixture, only in that case where the mixture is uniform. If, on the other hand, the molecules of the two gases possessed the same normal velocities (due to equality of molecular mass), there would be no dynamical cause for any particular mixture more than another, or every assigned mixture (regular or irregular) would be equally probable: for the distribution of the velocities would be symmetrical or uniform, whatever the mixture might be. Taking our illustration of the spherical portion of gas, and supposing the gas surrounding it (though chemically different) to have equal molecular velocity, then the exchange of molecules between the gases would take place at the same rate, and consequently there would be no disturbance of the equilibrium of pressure at all, but one mixture would be as possible as another, and the distribution of the velocities would be symmetrical whatever the mixture might be. ${ }^{2}$ In fact, it would resemble the case of the diffusion of two portions of one and the same gas into each other.

Thus it would appear that the fact of the molecules of the constituent gases of the atmosphere possessing unequal normal velocities (due to inequality of molecular mass) tends, through the dynamic action of the molecules, to produce and maintain forcibly a uniform mixture of these gases, and to prevent those detrimental irregularities of mixture that would inevitably occur (by a sufficient range of time and space), if the constituent gases of the atmosphere were of equal molecular masses, and consequently diffusion were brought under the pure contingencies of chance.

I have ventured to call attention to this point from its apparent importance, and as the passage above quoted would have the appearance at least of treating the problem as one of pure chances, or as if the influence of the in equality in molecular velocity had not been taken into account, but I shall be glad to accept correction if I am wrong.

The fact of the two gases of the atmosphere possessing snequal molecular masses would evidently seem to be of importance as a means for scattering and thereby rendering harmless, noxious vapours and gases which are emitted into the atmosphere. For even if the particular vapour (in a rare case) happened to be of the same molecular mass as one of the constituents of the atmosphere, it must differ from that of the other constituent, and thus a dynamical cause for dispersion exists. The considerable inequality in molecular mass of the most prevalent deleterious ingredient emitted in combustion and in the

$x$ The expansion may be seen by inclosing the oxygen in any elastic porous envelope, capable of expansion, and through which diffusion can freely take place. It may be observed that unmixed gases (of wneoual molecular masses, of course) are known to possess a capacity for 'work, which ceases the gases become uniformly mixed.

a It is conceivable that although the mean velucities or masses might be the same, the mean length of path of the molecules of the two gases might be slightly different. We must therefore either suppose a case where it is the same, or if minute exactness be desired, take it mto account. course of animal life (carbonic acid) thus ensures its dispersion.

In a paper published in the Phil. Mag. for April, 1875, by Lord Rayleigh-" On the Work that may be gained during the Mixing of Gases" -it was pointed out that work may be derived from gases in an unmixed state, and a special method for effecting this end was described. In two papers communicated by me to NATURE (vol. xvii. pp. $3 \mathrm{I}$ and 202), I, being at that time unaware of Lord Rayleigh's memoir, indicated a simple mechanical means of deriving work from unmixed gases by the use of porous diaphragms. If we imagine a cylinder, into the piston of which a disk or diaphragm of some porous substance (say plumbago) is fixed, and that two gases of unequal molecular masses (oxygen and hydrogen, for instance) are introduced into the opposite compartments of the cylinder, then diffusion commences in the known manner through the porous diaphragm. Owing to the inequality in the normal velocities of the molecules of the two gases, they pass through the pores of the diaphragm at unequal rates, thereby entailing an inequality of pressure on the two sides of the diaphragm. If then the piston (containing the diaphragm) be suddenly released, it will be driven towards the opposite end of the cylinder, and work may thus be derived. [A simple automatic device for continuing the work by a constant supply of gas was described in NATURE, vol. xvii. p. 204.] Although the work is here derived in a self-acting manner, solely at the expense of the normal temperature heat possessed by the gas, yet this would not apparently be out of harmony with the second law of thermodynamics [as the writer at first supposed]; for it appears that for such to be the case, the process would require to be a reversible one, or the gases would require to be restored again to their original unmixed state. But if it were possible that the gases could effect this themselves, or become unmixed by their mere action upon each other, and the probability, that in any assigned time we should find all the oxygen back again in the one half of the cylinder, where it was originally, and all the hydrogen back again in the other half, is one which can be measured (however remote this probability might be); then we should have a possible means of deriving work at the expense of normal temperature heat by a process that was self-reversible. Hence this result to which we are led would serve to confirm the above view, viz., that when gases are of unequal molecular masses, there is a forcible dynamical tendency to keep them mixed, or to prevent the gases from becoming separated. again when once they have become mixed.

There would seem to be another consideration bearing directly on the above case. It was a law enunciated by Dalton that when gases of different kinds are placed in the same vessel, "each gas behaves to the other as if it were a vacuum." This, when viewed by the light of the modern dynamical theory, is no doubt true as regards the fact that the total pressure on the sides of the vessel is the sum of the pressures which each gas would exert independently if placed by itself in the vessel. But if the expression "that one gas behaves to another as a vacuum" were taken to refer to the arrangement of the gas in the vessel, then some modification would appear to be required in the statement of the law in the case where the different gases are of equal molecular masses (as also where portions of gas of the same kind are successively introduced into the vessel). For it appears from the above considerations that gases do not necessarily become uniformly mixed by the action of diffusion, excepting when the gases are of wnequal molecular masses. For portions of gas of equal molecular masses behave to each other as portions of gas of the same kind. If we imagine (merely for illustration) the molecules of a portion of gas to be marked, and this portion of gas to be introduced into a vesscl where already gas of the same kind exists, then it is evident that there 
are an indefinite number of positions these marked molecules (representing the portion of introduced gas) could take up in the vessel, consistent with equilibrium, and there would be (practically) an infinite number of chances against the portion of introduced gas arranging itself as in a vacuum: for to do this, the marked molecules (composing the portion of gas) would require to arrange themselves in such a way that their mean distance is everywhere the same throughout the vessel, a contingency almost infinitely unlikely. What applies to marked molecules applies to chemically different molecules of equal mass, or which are dynamically similar. Hence it would follow that portions of gas of the same kind, or portions of chemically different gases of equal molecular mass could not be said "to behave to each other as vacua," in regard to arrangement. On the other hand, where the gases have unequal molecular masses there is (as we have seen) a forcible dynamical tendency for the gases to diffuse themselves symmetrically through each other, so that each gas behaves to the other as a vacuum, each gas becoming uniformly diffused through the ressel, as if it existed alone in a vacuum. The successive introduction into a vessel of portions of gas of the same kind (or of portions of chemically different gases of equal molecular mass) may be compared to the introduction into any closed space of successive sets of equal differently coloured perfectly elastic balls (the balls being supposed left in free motion among each other in analogy with the molecules of a gas), when evidently no one arrangement of the different coloured balls in the closed space (at any given instant) could be said to be more probable than another, and it would be extremely unlikely that the sets of coloured balls should "behave to each other as vacua," in the sense of each set diffusing itself symmetrically through the closed space, as it would do in a vacuum. But if the sets of balls were of unequal masses [in analogy to gases of unequal molecular masses], then no doubt the different sets would behave to each other as vacua, or each set would forcibly tend to arrange itself according to strict dynamical principles, so as to pervade uniformly the entire closed space, precisely as it would do a vacuum. S. TOLVER PRESTON

\section{OBSERVATIONS ON THE PHYSICAL GEO} GRAPHY AND GEOLOGY OF MADAGASCAR

A LTHOUGH Madagascar is known to be the third A largest island in the world, its actual size and extent is not very generally understood. It is easy to see how misconception on this point arises, for in maps the island is usually seen only in connection with Africa, and that great continent is so large that it dwarfs by comparison with itself everything in its near neighbourhood, so that the really large island sheltering under its southeastern side appears but an inconsiderable appendage to its vast neighbour. If, however, we take a good-sized map of Madagascar, and put by its side the outline, to the same scale, of another country with whose dimensions we are familiar, such, for instance, as England, we begin to realise how important an island it is as regards size, being nearly 1,000 miles long ${ }^{1}$ by about 250 in average breadth, so that it is nearly four times as large as England and Wales.

During the last ten years much light has been thrown upon the physical geography of Madagascar, principally through the researches of $\mathrm{M}$. Alfred Grandidier, and the numerous exploratory journeys made in various parts of the country by missionaries and others. Until a very recent period there was no reliable map of the island; a number of mountain ranges were shown in positions where no such geographical features are to be found, and the physical geography was completely misunderstood. But it is now quite clear that instead of a "central

$$
\text { x More exactly, } 975 \text { miles. }
$$

mountain chain," as described in most histories and gazetteers, there is an elevated mountainous region, which, however, does not occupy the centre of the island, but is more to the east and north, leaving a considerable extent of country to the west, and all beyond the $23 \mathrm{rd}$ parallel of south latitude, at a much lower level above the sea. Broadly speaking, therefore, Madagascar consists of two great divisions, viz., (I) an elevated interior region raised from 3,000 to 5,000 feet above the sea-level; and (2) a comparatively level country surrounding it, not much exceeding 400 or 500 feet in elevation, and most extensive in the west and south.

The elevated region is largely composed of primary and crystalline rocks. Lines of hills traverse it in all directions, but they do not rise to a very great height, the bighest points in the country, the peaks of the Ankàratra group of mountains, being a little under 9,000 feet above the sea-level. A very large extent of this portion of Madagascar is covered with bright red clay, through which the granite and basaltic rocks protrude. But there are also extensive rice-plains, especially near the capital cities of the two chief provinces, where there is a rich black alluvial soil ; and it can hardly be doubted that some at least of these plains, from their perfect level, out of which the red clay hills rise like islands, have formerly been the beds of extensive lakes, subsequently drained, possibly by slight changes in the level through subterranean action.

A good deal of this portion of Madagascar is bare and somewhat dreary-looking country. The long rolling moor-like hills are only covered with a coarse grass, which becomes very brown and dry towards the close of the seven months' rainless season ; but the hollows and rivervalleys are often filled with a luxuriant tropical vegetation, and, wherever there is population, with the bright green of the rice-fields. There is, nevertheless, an element of grandeur in the landscape, from the great extent of country visible from many points in the clear, pure atmosphere, which renders very distant objects wonderfully sharp and distinct. And many portions of the central region possess still greater claims to admiration from its picturesque mountain scenery.

In the southern Bétsiléo country, the grand and varied forms of the mountains filled me with an exultant kind of delight. To the south was a crowd of mountain-tops, peak beyond peak, with the greatest variety of outline : one had the appearance of a colossal truncated spire, another had a jagged saw-like ridge, another was like a pyramid with successive steps, and another an enormous dome. Their summits were never long free from clouds, and many of the peaks must be at least 3,000 feet above the plain.

Sections taken by the aneroid across this elevated region from east to west at the latitude of the capital show that it has a depression in the centre, the edges on either side being considerably higher than the country between them. At some points this height of 4,000 to 5,000 feet is gained by a series of steps from the maritime plains, each range of hills rising higher and higher, while at other points it descends almost at one steep slope for nearly 3,000 feet. The water-shed is not in the centre of the island, but is much nearer the eastern side. Through the eastern wall many of the rivers cut their way by magnificent gorges, amidst dense forest, finding their way to the sea by a succession of rapids and cataracts, and occasionally by stupendous falls, as in the case of the Matitanana river, which descends at one plunge 500 or 600 feet. Some of the western rivers, also, are said to form grand waterfalls, particularly that of the Mania, whose sound is reported to be heard at a distance of two days' journey, i.e., about forty to fifty miles.

The lower region of Madagascar consists of extensive plains only a few hundred feet above the sea-level, but there are at least three prominent chains of hills traversing it from north to south, one of which appears nearly 